\title{
Quasifission dynamics in TDHF
}

\author{
A.S. Umar ${ }^{1}$, V.E. Oberacker ${ }^{1}$ and C. Simenel ${ }^{2}$ \\ ${ }^{1}$ Department of Physics and Astronomy, Vanderbilt University, Nashville, \\ TN 37235, USA \\ ${ }^{2}$ Department of Nuclear Physics, RSPE, Australian National University, \\ Canberra, ACT 0200, Australia
}

\begin{abstract}
For light and medium mass systems the capture cross-section may be considered to be the same as that for complete fusion, whereas for heavy systems leading to superheavy formations the evaporation residue cross-section is dramatically reduced due to the quasifission (QF) and fusion-fission processes thus making the capture cross-section to be essentially the sum of these two cross-sections, with QF occurring at a much shorter time-scale. Consequently, quasifission is the primary reaction mechanism that limits the formation of superheavy nuclei. Within the last few years the time-dependent Hartree-Fock (TDHF) approach has been utilized for studying the dynamics of quasifission. The study of quasifission is showing a great promise to provide insight based on very favorable comparisons with experimental data. In this article we will focus on the TDHF calculations of quasifission observables for the ${ }^{48} \mathrm{Ca}+{ }^{249} \mathrm{Bk}$ system.
\end{abstract}

\section{Introduction}

One of the most fascinating research areas involving low-energy nuclear reactions is the search for superheavy elements. Experimentally, two approaches have been used for the synthesis of these elements, one utilizing targets in the lead region (cold-fusion) $[1,2]$, the other utilizing deformed actinide targets with ${ }^{48} \mathrm{Ca}$ projectiles (hot-fusion) [3-5]. While both methods have been successful in synthesizing new elements the evaporation residue cross-sections of 
the hot-fusion reactions were found to be as much as three times larger than those of the cold fusion ones. To pinpoint the root of this difference it is important to understand the details of the entrance channel dynamics of these systems since the properties of the dinuclear system at the capture point will strongly influence the outcome of the reaction. For light and medium mass systems the capture cross-section may be considered to be the same as that for complete fusion, whereas for heavy systems leading to superheavy formations the evaporation residue cross-section is dramatically reduced due to the quasi-fission [6] and fusion-fission processes, thus making the capture cross-section to be essentially the sum of these two cross-sections. What is also difficult to ascertain is the configuration of the composite system, namely, whether the system has a single-center compound-like configuration or a dinuclear configuration accompanied by particle exchange. Most dynamical models [7-11] argue that for heavy systems a dinuclear complex is formed initially and the barrier structure and the excitation energy of this precompound system will determine its survival to breaking up via quasifission. Furthermore, if the nucleus survives this initial state and evolves to a compound system it can still fission due to its excitation.

Within the last few years the time-dependent Hartree-Fock (TDHF) approach $[12,13]$ has been utilized for studying the dynamics of quasifission [14-18] and scission dynamics [19-21]. Particularly, the study of quasifission is showing a great promise to provide insight based on very favorable comparisons with experimental data. Similarly, an extension of TDHF called the density-constrained TDHF [22] (DC-TDHF) has been used to obtain microscopic potential barriers and capture cross-sections for superheavy [23] and lighter systems [24-26]. In this article we will focus on the TDHF studies of quasifission for the ${ }^{48} \mathrm{Ca}+{ }^{249} \mathrm{Bk}$ system.

\section{Results}

During the past several years it has become feasible to perform TDHF calculations on a three-dimensional (3D) Cartesian grid with no symmetry restrictions and with much more accurate numerical methods $[27,28]$. In the present TDHF calculations we use the Skyrme SLy4d energy density functional (EDF) [29] including all of the relevant time-odd terms in the meanfield Hamiltonian. First we generate very accurate static HF wave functions for the two nuclei on the 3D grid. The initial separation of the two nuclei is $30 \mathrm{fm}$. In the second step, we apply a boost operator to the single-particle wave functions. The time-propagation is carried out using a Taylor series 
expansion (up to orders $10-12$ ) of the unitary mean-field propagator, with a time step $\Delta t=0.4 \mathrm{fm} / \mathrm{c}$. By virtue of long contact-times for quasifission and the energy and impact parameter dependence these calculations require extremely long CPU times. In Fig. 1a we plot the microscopic DC-TDHF
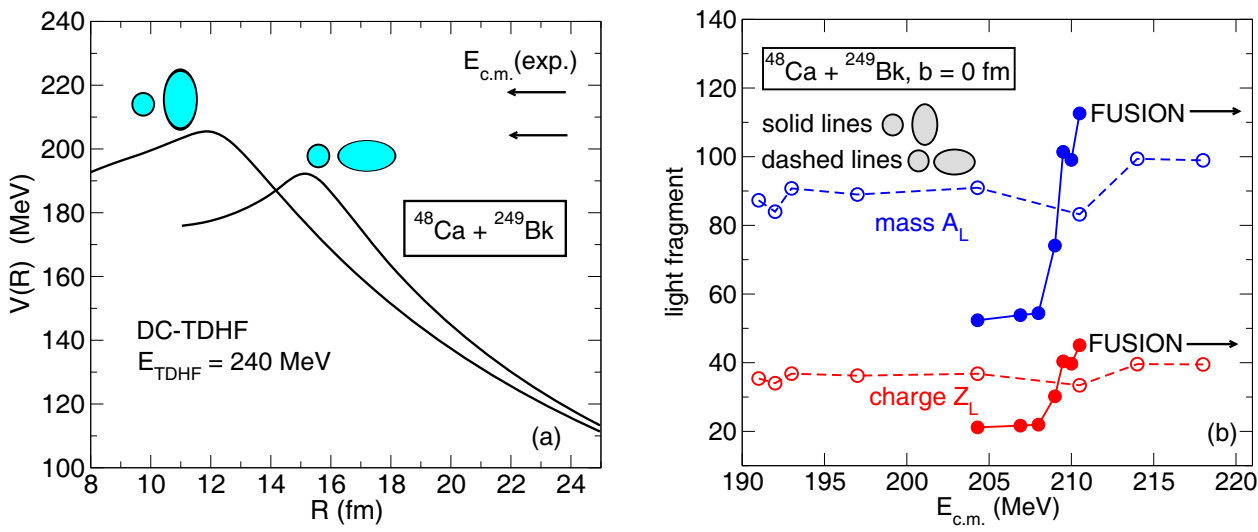

Figure 1: (a) Nucleus-nucleus potential, $V(R)$, for the ${ }^{48} \mathrm{Ca}+{ }^{249} \mathrm{Bk}$ system obtained from DC-TDHF calculation for selected orientation angles of the ${ }^{249} \mathrm{Bk}$ nucleus. Also shown are the experimental c.m. energies. (b) Mass and charge of the light fragment as a function of $E_{\text {c.m. }}$. for central collisions of ${ }^{48} \mathrm{Ca}$ with the side and tip orientations of ${ }^{249} \mathrm{Bk}$.

potential barriers obtained for the ${ }^{48} \mathrm{Ca}+{ }^{249} \mathrm{Bk}$ system. The two barriers depict the two extreme orientations of the ${ }^{249} \mathrm{Bk}$ nucleus. Also, shown are the experimental energies at which this reaction has been studied [4,30]. As expected the polar or tip orientation of ${ }^{249} \mathrm{Bk}$ results in a significantly lower barrier. The highest experimental energy is above both barriers but the lowest experimental energy is below the barrier for the equatorial or side orientation of ${ }^{249} \mathrm{Bk}$. Figure $1 \mathrm{~b}$ shows the mass and charge of the light fragment as a function of $E_{\text {c.m. }}$. for central collisions of ${ }^{48} \mathrm{Ca}$ with the side and tip orientation of ${ }^{249} \mathrm{Bk}$. For the side orientation of ${ }^{249} \mathrm{Bk}$ for energies below $E_{\text {c.m. }}=204 \mathrm{MeV}$ we get quasielastic collisions whereas for energies above $211 \mathrm{MeV}$ we see fusion, which we define arbitrarily as reactions with contact times exceeding 35 zs. Naturally, non-central impact parameters can show quasifission in the range where we see fusion. We observe that for central collisions with the side orientation of ${ }^{249} \mathrm{Bk}$ quasifission is limited to a small range of energies $E_{\text {c.m. }}=209-211 \mathrm{MeV}$. The quasifission results are very different for the tip orientation of ${ }^{249} \mathrm{Bk}$, ranging over a much wider energy domain, from $E_{\text {c.m. }}=191 \mathrm{MeV}$ to $E_{\text {c.m. }}=218 \mathrm{MeV}$, which is the highest energy we have computed. The tip orientation also leads to smaller 
maximum mass and charge transfer.
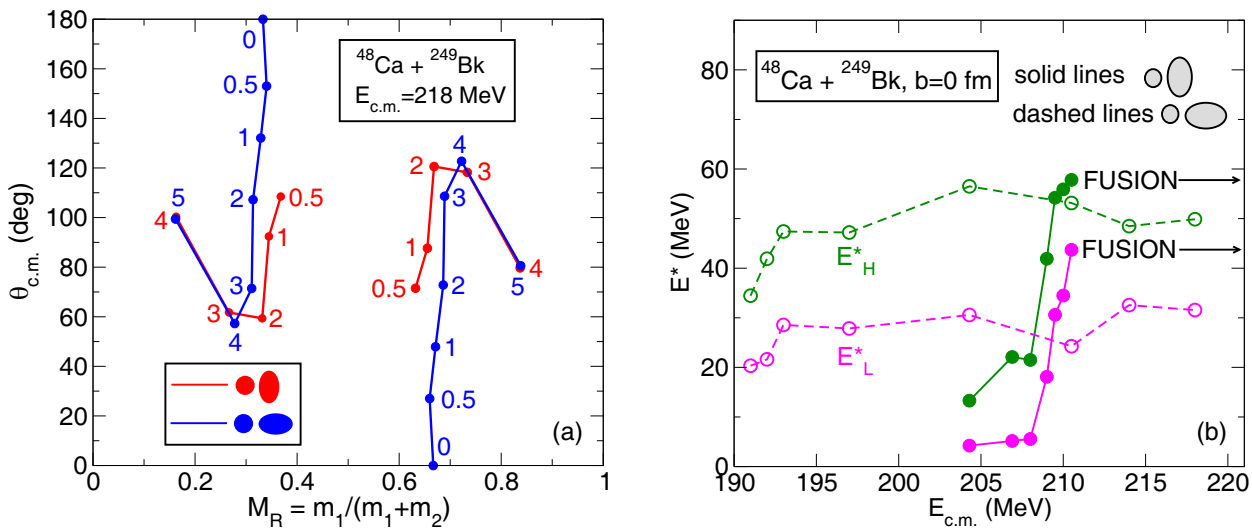

Figure 2: (a) Mass-angle distribution for the ${ }^{48} \mathrm{Ca}+{ }^{249} \mathrm{Bk}$ system for the tip and side orientations of the ${ }^{249} \mathrm{Bk}$ nucleus. (b) Excitation energy, $E^{*}$, of the heavy and light fragments as a function of $E_{\text {c.m. }}$. for central collisions and for two orientations of the ${ }^{249} \mathrm{Bk}$ nucleus.

In Fig. 2(a) we show the TDHF calculations of quasifission MADs for ${ }^{48} \mathrm{Ca}+{ }^{249} \mathrm{Bk}$ at $E_{\text {c.m. }}=218 \mathrm{MeV}$, corresponding to the two orientations of the ${ }^{249} \mathrm{Bk}$ nucleus. The regions of MAD's near $M_{R}=0.2$ and $M_{R}=0.8$ correspond to elastic and quasielastic reactions, followed by transition to deep-inelastic reactions and subsequently quasifission. The TDHF calculations predict where the transition from deep-inelastic to quasifission occurs, as well as the general behavior of the MADs. However, due to the fact that TDHF is a deterministic theory, it will only give us the most probable outcome or path for the MADs rather than a full distribution.

Recently, we have developed an extension to TDHF theory via the use of a density constraint to calculate the excitation energy of each fragment directly from the TDHF density evolution. This gives us new information on the repartition of the excitation energy between the heavy and light fragments which is not available in standard TDHF calculations. In Fig. 2b we show that the heavy and light fragments contain up to $60 \mathrm{MeV}$ and $45 \mathrm{MeV}$ (side orientation) and up to $\sim 55 \mathrm{MeV}$ and $\sim 30 \mathrm{MeV}$ (tip orientation) of excitation energy, respectively. It is interesting to note that the excitation energy follows qualitatively the mass and charge transfer behaviour observed in Fig. 1-b.

Figure 3a shows the contact time as a function of impact parameter at $E_{\text {c.m. }}=218 \mathrm{MeV}$. We see that the contact times are 8-30 zs for impact parameters resulting in quasifission and falls sharply for fragments produced in 
deep-inelastic collisions. These contact times are in agreement with typical quasi-fission times obtained from interpretations of experimental mass-angle distributions of the fragments $[6,31,32]$.

Figure $3 \mathrm{~b}$ show the mass and charge of the light fragment for the ${ }^{48} \mathrm{Ca}+{ }^{249} \mathrm{Bk}$ system as a function of impact parameter for the two orientations of the ${ }^{249} \mathrm{Bk}$ nucleus calculated at $E_{\text {c.m. }}=218 \mathrm{MeV}$. As expected quasifission is identified with large mass and charge transfer, in this case corresponding to the doubling of the charge from 20 to 40 and mass from 48 to 100 . It is also interesting to note the slightly atypical value of the contact time at impact parameter $b=2 \mathrm{fm}$ in Fig. 3a in comparison to the neighboring impact parameters. Fig. $3 \mathrm{~b}$ shows that in this region the light fragment is a neutron rich $\mathrm{Zr}$ isotope with $A \approx 102-106$. The microscopic evolution of the shell structure seems to have a tendency to form a composite with a longer lifetime when the light fragment is in this region. Similar observations were made in ${ }^{40,48} \mathrm{Ca}+{ }^{238} \mathrm{U}$ quasifission study [17]. A possible explanation is the presence of strongly bound deformed isotopes of $\mathrm{Zr}$ in this region $[33,34]$.
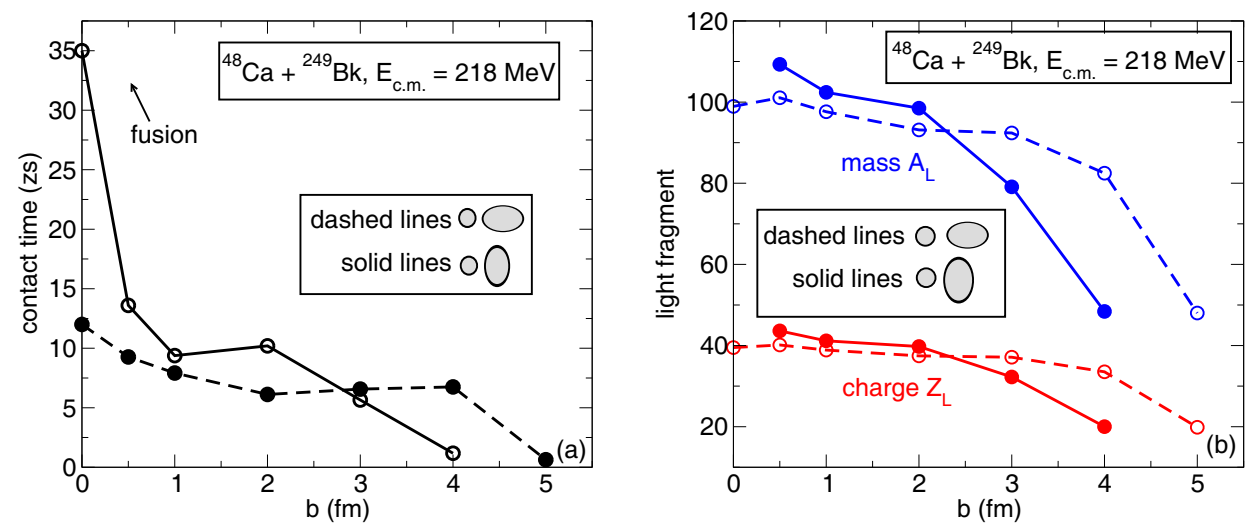

Figure 3: (a) Contact time and (b) mass and charge of the light fragment for the ${ }^{48} \mathrm{Ca}+{ }^{249} \mathrm{Bk}$ system as a function of impact parameter for the two orientations of the ${ }^{249} \mathrm{Bk}$ nucleus calculated at the c.m. energy of $E_{\text {c.m. }}=218 \mathrm{MeV}$.

\section{Summary}

We have shown recent quasifission results for the ${ }^{48} \mathrm{Ca}+{ }^{249} \mathrm{Bk}$ system. Further calculations are underway to obtain a full range of observables including mass-angle distributions and fragment TKE's. Recent TDHF calculations of phenomena related to superheavy element searches show that TDHF can 
be a valuable tool for elucidating some of the underlying physics for these reactions. As a microscopic theory with no free parameters, where the effective nucleon-nucleon interaction is only fitted to the static properties of a few nuclei, these results are very promising.

\section{Acknowledgments}

This work has been supported by the U.S. Department of Energy under grant No. DE-SC0013847 with Vanderbilt University and by the Australian Research Council Grant No. FT120100760.

\section{References}

[1] S. Hofmann and G. Münzenberg, Rev. Mod. Phys., 72 (2000) 733.

[2] S. Hofmann, F. P. Heßberger, D. Ackermann, G. Münzenberg, S. Antalic, P. Cagarda, B. Kindler, J. Kojouharova, M. Leino, B. Lommel, R. Mann, A. G. Popeko, S. Reshitko, S. Śaro, J. Uusitalo, and A. V. Yeremin, Eur. Phys. J. A, 14 (2002) 147.

[3] Yuri Oganessian, J. Phys. G, 34 (2007) R165.

[4] Yu. Ts. Oganessian, F. Sh. Abdullin, C. Alexander et al., Phys. Rev. C, 87 (2013) 054621.

[5] S. Hofmann, D. Ackermann, S. Antalic et al., Eur. Phys. J. A, 32 (2007) 251.

[6] J. Töke and R. Bock and G.X. Dai et al., Nucl. Phys. A 440 (1985) 327.

[7] G. Fazio, G. Giardina, G. Mandaglio, R. Ruggeri et al., Phys. Rev. C, 72 (2005) 064614.

[8] G. G. Adamian, N. V. Antonenko, and W. Scheid, Phys. Rev. C, 68 (2003) 034601.

[9] G. G. Adamian, N. V. Antonenko, and W. Scheid, Eur. Phys. J. A, 41 (2009) 235.

[10] A. K. Nasirov, G. Giardina, G. Mandaglio et al., Phys. Rev. C, 79 (2009) 024606. 
[11] Zhao-Qing Feng, Gen-Ming Jin, Jun-Qing Li, and Werner Scheid, Nucl. Phys. A, 816 (2009) 33.

[12] J. W. Negele, Rev. Mod. Phys., 54 (1982) 9135.

[13] C. Simenel, Eur. Phys. J. A, 48 (2012) 152.

[14] C. Golabek and C. Simenel, Phys. Rev. Lett., 103 (2009) 042701.

[15] D. J. Kedziora and C. Simenel, Phys. Rev. C, 81 (2010) 044613.

[16] A. Wakhle, C. Simenel, D. J. Hinde et al., Phys. Rev. Lett., 113 (2014) 182502 .

[17] V. E. Oberacker, A. S. Umar, and C. Simenel, Phys. Rev. C, 90 (2014) 054605 .

[18] K. Hammerton, Z. Kohley, D. J. Hinde et al., Phys. Rev. C, 91 (2015) 041602(R).

[19] C. Simenel and A. S. Umar, Phys. Rev. C, 89 (2014) 031601.

[20] G. Scamps, C. Simenel, and D. Lacroix, Phys. Rev. C, 92 (2015) 011602.

[21] P. M. Goddard, P. D. Stevenson, and A. Rios, arXiv:1504.00919, (2015).

[22] A. S. Umar and V. E. Oberacker, Phys. Rev. C, 74 (2006) 021601.

[23] A. S. Umar, V. E. Oberacker, J. A. Maruhn, and P.-G. Reinhard, Phys. Rev. C, 81 (2010) 064607.

[24] V. E. Oberacker, A. S. Umar, J. A. Maruhn, and P.-G. Reinhard, Phys. Rev. C, 82 (2010) 034603.

[25] R. Keser, A. S. Umar, and V. E. Oberacker, Phys. Rev. C, 85 (2012) 044606 .

[26] C. Simenel, R. Keser, A. S. Umar, and V. E. Oberacker, Phys. Rev. C, 88 (2013) 024617.

[27] C. Bottcher, M. R. Strayer, A. S. Umar, and P.-G. Reinhard, Phys. Rev. A, 40 (1989) 4182; Phys. Rev. A, 41 (1990) 1399.

[28] J. A. Maruhn, P.-G. Reinhard, P. D. Stevenson, and A. S. Umar, Comp. Phys. Comm., 185 (2014) 2195. 
[29] Ka-Hae Kim, Takaharu Otsuka, and Paul Bonche, J. Phys. G, 23 (1997) 1267.

[30] J. Khuyagbaatar, A. Yakushev, Ch. E. Düllmann et al., Phys. Rev. Lett., 112 (2014) 172501.

[31] R. du Rietz, D. J. Hinde, M. Dasgupta et al., Phys. Rev. Lett. 106 (2011) 052701.

[32] R. du Rietz, E. Williams, D. J. Hinde et al., Phys. Rev. C 88 (2013) 054618 .

[33] V. E. Oberacker, A. S. Umar, E. Terán, and A. Blazkiewicz, Phys. Rev. C, 68 (2003) 064302.

[34] A. Blazkiewicz, V. E. Oberacker, A. S. Umar, and M. Stoitsov, Phys. Rev. C, 71 (2005) 054321. 